\title{
Infinite graph embeddings on tubular surfaces
}

\author{
Boza, L. ${ }^{\text {a }}$ Fedriani, E.M. ${ }^{\text {b }}$, Márquez, A. ${ }^{\text {, }}$ \\ Núñez, J. ${ }^{\mathrm{d}}$ and Revuelta, P. ${ }^{\mathrm{e}}$ \\ ${ }^{a}$ Departamento de Matemática Aplicada I. Escuela Técnica Superior de \\ Arquitectura. Universidad de Sevilla. \\ ${ }^{\mathrm{b}}$ Departamento de Economía y Empresa. Facultades de Ciencias Empresariales y \\ Experimentales. Universidad Pablo de Olavide. \\ ${ }^{c}$ Departamento de Matemática Aplicada I. Facultad de Informática y Estadística. \\ Universidad de Sevilla. \\ 'Departamento de Geometría y Topología. Facultad de Matemáticas. Universidad \\ de Sevilla. \\ ${ }^{\mathrm{e}}$ Departamento de Matemática Aplicada I. Escuela Universitaria de Arquitectura \\ Técnica. Universidad de Sevilla.
}

\section{Introduction}

The problem of extending Kuratowski's planarity criterium [7] to other surfaces appers to be very difficult, and there was little progress on this problem until 1979, when Archdeacon, Glover, Huneke and Wang [1,5] determined the class of all graphs which cannot be drawn in $S$ and which are minimal with this property under topological containment -denoted by $T(S)$ - when $S$ is the projective plane. They found that in that case $T(S)$ has 103 members.

About the planarity of infinite graph, Dirac and Schuster [4] proved that a countable graph is planar if and only if each finite subgraph is planar, and Wagner in [12] characterized all planar graphs. But, as many authors as Halin or Thomassen (see [6,11]) have pointed out, some additional properties can be, and must be, added to planarity in the case of infinite graphs. In particular, from a practical point of view, accumulation points must be avoided.

Halin gave in $[6]$ the characterization of the graphs with an embedding without vertex accumulation point in terms of forbidden subgraphs.

In 1994, Thomassen proposed us the generalization of Halin's theorem to the cylinder. The characterization of the graphs that admit embeddings with no vertex accumulation point on tubular surfaces of finite genus is found in [8]. This characterization is get as a consequence of a relation between two families of graphs. One of those families is the set infinite countable locally finite graphs with an embedding in $S(n)$ with no vertex accumulation point; and the other family is the set of finite graphs with an embedding in the compact surface $S$ 


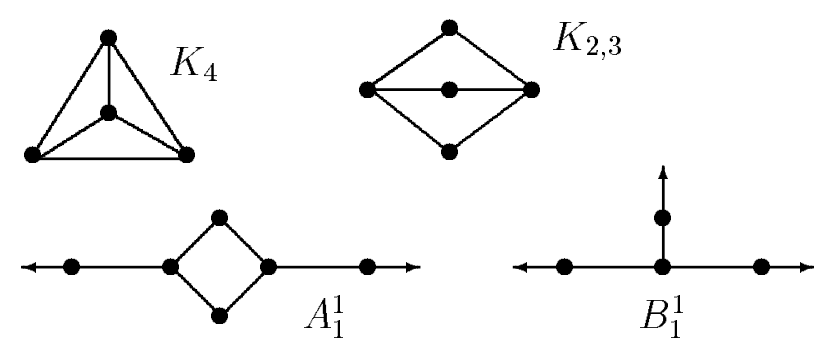

Fig. 1. Forbidden p-outerplanar minors.

such that the vertices of a subset of their vertices are in $n$ faces. As another consequence of that relation and the important result about graph minors obtained by Robertson and Seymour in [9], it is proved that the minimal set of forbidden minors for the graph embeddings with no vertex accumulation point in non-compact surfaces is finite.

On the other hand, the finite outerplanar graphs characterization, by Chartrand and Harary in [3], is also valid for infinite countable graphs: a graph admits an embedding in the plane with all its vertices in the same face if and only if it does not have a subgraph which is a subdivision of either $K_{4}$ or $K_{2,3}$. However, in order to assure that a graph has an outerplanar embedding without accumulation points of either vertices or edges (as is suggested in [10]), such a graph needed to be locally finite and not have two other forbidden minors more, according to the following result, by Boza, Diánez and Márquez $[2]:$

An infinite countable graph is p-outerplanar if and only if it does not have a minor among $K_{4}, K_{2,3}, A_{1}^{1}$ or $B_{1}^{1}$ (see Figure 1 ).

\section{Main Results}

The main goal of this paper is double: on the one hand, we show a characterization of the graph embeddings with no accumulation point on Möbius Band in terms of forbidden minors. On the other hand, we give the list of forbidden p-outercyilindrical minors. These results are the following:

\section{Theorem 1}

A graph has an embedding without vertex accumulation point on Möbius Band if and only if it does not contain a minor from a list with 291 graphs.

\section{Theorem 2}

A graph has an embedding without edges accumulation point on Möbius Band if and only if it does not contain a minor from a list with 293 graphs.

\section{Theorem 3}

A graph is p-outercyilindrical if and only if it does not contain a minor from the following list: $K_{4}, K_{2,3}, A_{1}^{1} \cup A_{1}^{1}, A_{1}^{1} \cup B_{1}^{1}, B_{1}^{1} \cup B_{1}^{1}, M_{i}$, with $i=1, \ldots, 6$ (see Figures 1 and 2). 

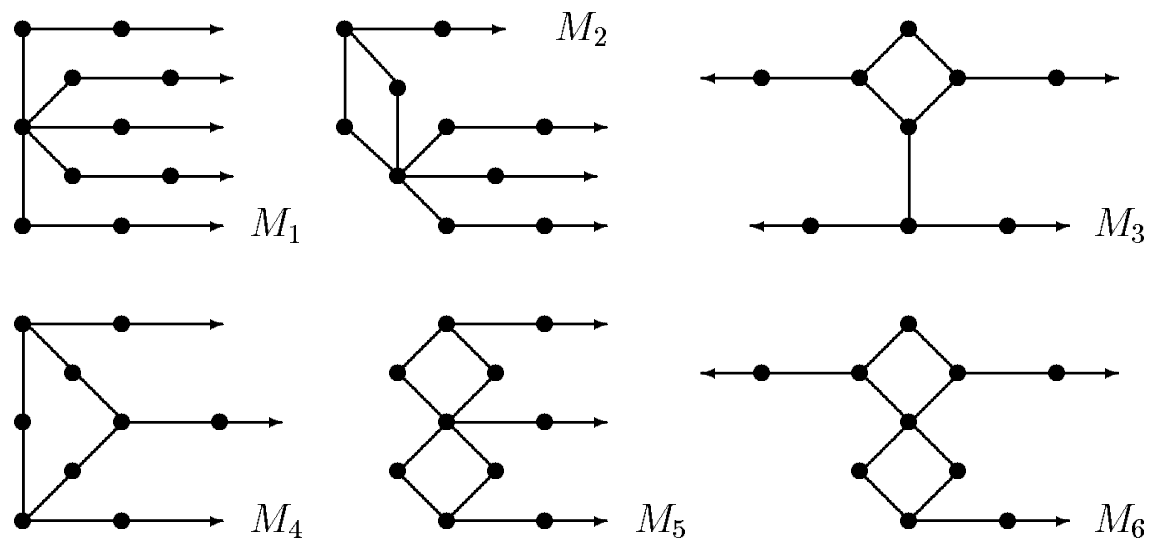

Fig. 2. Forbidden p-outercylindrical minors.

\section{References}

[1] D. Archdeacon. A Kuratowski Theorem for the Projective Plane. Tesis, The Ohio State University, 1980.

[2] Boza, L; Diánez, A; Márquez, A. "On infinite outerplanar graphs". Mathematica Bohemica 119:4, (1994), 381-384.

[3] Chartrand, G; Harary, F. "Planar permutation graphs". Ann. Inst. H. Poincaré. Sect. B. 3 (1967), 433-438.

[4] G. A. Dirac y S. Schuster. A Theorem of Kuratowski. Indag Math., 16: 343-348, 1954.

[5] H. H. Glover, J. P. Huneke y C. S. Wang. 103 graphs that are irreducible for the projective plane. Journal of Combinatorial Theory 27(3): 332-370, 1979.

[6] H. Halin. Zur häufungspunktfreien Darstellung abzählbarer Graphen in der Ebene. Arch. Math., 17: 239-242, 1966.

[7] K. Kuratowski. Sur le problème des courbes gauches en topologie. Fund. Math. 5: 271-283, 1930.

[8] Revuelta, M. P. "Inmersiones de grafos en superficies tubulares de género finito". Ph. D. Thesis. Dpto. de Matemática Aplicada I. Univ. de Sevilla (Spain), 1999.

[9] N. Robertson y P.D. Seymour. Graph Minors VIII. A Kuratowski Theorem for General Surfaces. J. Combinatorial Theory. Serie B., 48: 255-288, 1990.

[10] Thomassen, C. "Infinite Graphs". Selected Topics in Graph Theory. 2 Academic Press (1983), 129-160.

[11] C. Thomassen. Straight line representations of infinite planar graphs. J. London Math. Soc., 16(2): 411-423, 1977.

[12] K. Wagner. Bemerkungen zum Vierfarbenproblem. Jahresber. Deutsch. MathVerein. 46: 26-32, 1936. 